\title{
Permanent magnet trap for cold atoms
}

\author{
J. J. Tollett, C. C. Bradley, C. A. Sackett, and R. G. Hulet \\ Physics Department and Rice Quantum Institute, Rice University, Houston, Texas 77251-1892
}

(Received 19 September 1994)

\begin{abstract}
We report the trapping of neutral atoms in a permanent magnet trap. Approximately $1 \times 10^{8}$ ground-state lithium atoms have been confined in a nonzero magnetic-field minimum produced by six permanent magnets in an Ioffe configuration. These atoms have a kinetic temperature of $1.1 \mathrm{mK}$ and a peak density of approximately $3 \times 10^{9} \mathrm{~cm}^{-3}$. The trapped-atom lifetime is $240 \mathrm{~s}$, limited by collisions with background gas. This trap provides an environment in which quantum-statistical effects, atomic collisions, and other ultralow-temperature phenomena can be studied.
\end{abstract}

PACS number(s): 32.80.Pj, 03.75.Fi

The past decade has seen steady progress in neutral-atom trapping with a resulting improvement in the production of dense, ultracold atomic vapors. These vapors have been useful for both high-resolution spectroscopy and investigations of ultracold atom collisions [1]. Further significant increases in cold-atom densities will allow the observation and study of quantum-statistical effects of weakly interacting particles, such as Bose-Einstein condensation and anomalous light scattering. We report a significant advance in neutral-atom trapping by confining spin-polarized ${ }^{7} \mathrm{Li}$ atoms in a permanent magnet trap. Permanent magnet traps have been proposed [2] as a means for providing tightly confining potentials helpful in achieving high atomic densities [3]. The use of permanent magnets can produce high field strengths and gradients comparable to those of superconducting magnet traps but with reduced complexity and greater optical access.

Recently, a magneto-optical trap using two permanent magnet disks to produce the required quadrupole field was demonstrated [4] and a few atoms were observed to remain trapped around the magnetic field zero when the trap laser beams were blocked. The permanent magnet trap described here uses an arrangement of magnets in an Ioffe configuration [5], which features a nonzero magnetic-field minimum. Atoms are trapped about this minimum through the interaction of their total magnetic moment with the trap's magnetic field. The nonzero minimum prevents trap-loss-producing spin-flip (Majorana) transitions, which can occur as atoms pass through or near a field zero [6,7].

As shown in Fig. 1, the trap consists of six axially magnetized, cylindrical, permanent magnets symmetrically positioned and aligned along three mutually orthogonal axes. The four magnets in the $x-y$ plane are oriented to produce a quadrupole field, while the two magnets along the $z$ axis generate a dipole field. For atoms in the proper spin state, the resulting potential has a minimum at the trap center and has relatively low potential barriers, or "thresholds," along the four axes that pass through the center and between magnet tips of like sign. The magnets are supported by a nickel-plated iron yoke that recycles the magnetic flux, helping to increase the bias field and field gradients. The magnets are nickel-plated, 27MG Oe energy-product, neodymium-iron-boron (Nd-Fe-B) magnets, measuring $2.54 \mathrm{~cm}$ long by $2.22 \mathrm{~cm}$ diameter and positioned in the yoke with a tip-to-tip spacing of $4.45 \mathrm{~cm}$.
The nickel plating of the magnets and the yoke prevents corrosion and provides ultrahigh-vacuum compatibility. NdFe-B can have a remnant magnetization as high as $12 \mathrm{kG}$, and with an intrinsic coercivity of $18 \mathrm{kOe}$ is highly resistant to demagnetization. Each trap magnet has a surface magnetization of $5 \mathrm{kG}$. By comparison, a coil of like dimensions must carry nearly $9 \mathrm{kA}$ turns of current to generate this field. Such a current density is impractical for conventional conductors. Superconducting coils can easily generate current densities of this magnitude, but at the cost of increased size and complexity.

The high intrinsic coercivity of the Nd-Fe-B magnets allows the fields to be accurately calculated with a simple model. By assuming a uniform magnetization $\mathbf{M}$ directed along the magnet axis, each magnet can be modeled by a uniform magnetic pseudo-surface-charge density on its inner face of $\hat{\mathbf{n}} \cdot \mathbf{M}$, where $\hat{\mathbf{n}}$ is the outward normal of the magnet. The outer faces of the magnets make a negligible contribution to the trap field because of the presence of the yoke. The scalar potential $\varphi(\mathbf{r})$ and magnetic field $\mathbf{B}(\mathbf{r})$ at a position $\mathbf{r}$ in the trapping region are given by

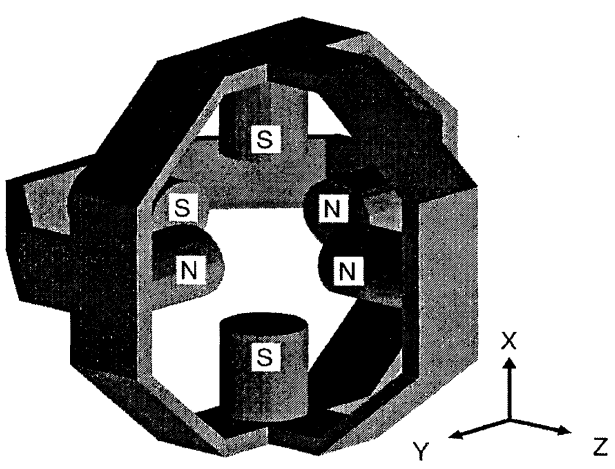

FIG. 1. Diagram showing the orientation and placement of the cylindrical trap magnets for the permanent magnet, Ioffe configuration trap. The letters indicate the inner tip magnetizations of the $\mathrm{Nd}-\mathrm{Fe}-\mathrm{B}$ cylinder magnets, $\mathrm{N}$ for north and $\mathrm{S}$ for south. The tip-totip magnet spacing is $4.45 \mathrm{~cm}$. The structure around the magnets is an iron yoke that supports the magnets and provides low reluctance paths for the flux to follow between magnets of opposite sign. 


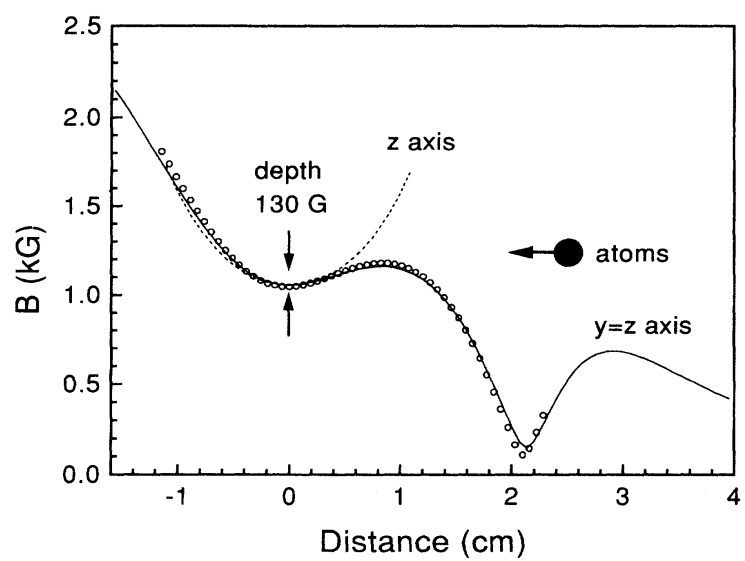

FIG. 2. Calculated trap field magnitude $B$ along the $y=z$ (solid) and $z$ (dashed) axes, and measured field along the $y=z$ axis (circles). In the experiment, the atoms enter along the $y=z$ axis from the right and are illuminated by the trap laser beams beginning near the threshold field. The atoms are slowed along the loading path, then damped about the trap center by the optical molasses formed by the trap beams. Once trapped they are held in a 130-Gdeep well, corresponding to a depth of $9 \mathrm{mK}$ for ${ }^{7} \mathrm{Li}$.

$$
\mathbf{B}(\mathbf{r})=-\mu_{0} \nabla \varphi(\mathbf{r})=-\mu_{0} \nabla\left(\sum_{i=1}^{6} \frac{1}{4 \pi} \int_{S_{i}} \frac{\hat{\mathbf{n}}_{i} \cdot \mathbf{M}_{i}}{\left|\mathbf{r}-\mathbf{r}^{\prime}\right|} d a^{\prime}\right),
$$

where the sum accounts for contributions from the six magnet surfaces $S_{i}$, and $\mu_{0}$ is the permeability of free space. Prior to installing the trap assembly into the vacuum apparatus the magnetic fields were mapped using a miniature threeaxis Hall probe. Figure 2 shows a plot of the calculated magnetic field strength $B=|\mathbf{B}|$ along the $z$ axis and one of the four threshold axes. This plot demonstrates that the model results agree well with the measured values of the field. The bias field of $1050 \mathrm{G}$ and threshold of $1180 \mathrm{G}$ determine the trap well depth to be $130 \mathrm{G}$, corresponding to $9 \mathrm{mK}$ for ${ }^{7} \mathrm{Li}$. Near the trap center, the field magnitude is essentially parabolic with measured harmonic coefficients of $d^{2} B / d x^{2}=d^{2} B / d y^{2}=950 \quad \mathrm{G} / \mathrm{cm}^{2}, \quad$ and $d^{2} B / d z^{2}=860$ $\mathrm{G} / \mathrm{cm}^{2}$. The corresponding oscillation frequencies for ${ }^{7} \mathrm{Li}$ are $v_{x}=v_{y}=140 \mathrm{~s}^{-1}$ and $v_{z}=130 \mathrm{~s}^{-1}$.

$\mathrm{Nd}-\mathrm{Fe}-\mathrm{B}$ magnets have a Curie temperature of $300^{\circ} \mathrm{C}$ but can be partially demagnetized at significantly lower temperatures, depending on the grade of magnetic material. We found that after an excessively hot vacuum bakeout, at temperatures near $150{ }^{\circ} \mathrm{C}$, the trap bias field had suffered a $20 \%$ reduction. We believe this demagnetization could have been prevented by limiting the bakeout temperature below $125^{\circ} \mathrm{C}$. The trap has nevertheless been successfully used to trap atoms, as will be described.

The trap is loaded along a threshold axis with cold atoms produced by slowing the low-velocity fraction of a thermal atomic beam. Atoms with velocities less than $500 \mathrm{~m} / \mathrm{s}$ are slowed to an average velocity of $50 \mathrm{~m} / \mathrm{s}$ by a $20-\mathrm{cm}-$ long, $\sigma^{-}$-type Zeeman slower [8]. A cylindrically focused traveling-wave laser beam is used to deflect the slow atoms by an angle of $30^{\circ}$ from the fast part of the atomic beam and toward the trap [9]. The deflected atoms are collimated and optically pumped into the trappable ground state by twodimensional optical molasses [10]. In the trap, a fraction of these atoms is captured and cooled by optical molasses [11] provided by three pairs of $1-\mathrm{cm}$-diam, retroreflected laser beams. The total intensity at the trap center is $25 \mathrm{~mW} / \mathrm{cm}^{2}$. These trap beams are tuned near the resonance frequency of a cooling transition at the magnetic field minimum and are directed along three of the four body diagonals of the cube defined by the inner faces of the trap magnets. The required three-dimensional damping is facilitated by the good optical access to the trap center.

Atoms are trapped in the $2 S_{1 / 2}\left(m_{J}=\frac{1}{2}, m_{I}=\frac{3}{2}\right)$ state, where the quantum numbers $m_{J}$ and $m_{I}$ are the projections of the total electronic and nuclear angular momenta, respectively, along the local magnetic-field direction. The cooling transition used is the $2 S_{1 / 2}\left(m_{J}=\frac{1}{2}, m_{I}\right.$ $\left.=\frac{3}{2}\right) \leftrightarrow 2 P_{3 / 2}\left(m_{J}=\frac{3}{2}, m_{I}=\frac{3}{2}\right)$ transition which forms a cycling, two-level system when excited by $\sigma^{+}$polarized light. This transition has a linewidth of $5.9 \mathrm{MHz}$, a saturation intensity of $5.1 \mathrm{~mW} / \mathrm{cm}^{2}$, and a wavelength of $671 \mathrm{~nm}$. Given the need for three-dimensional damping, it is impossible to provide pure $\sigma^{+}$-polarized light. Due to the presence of $\pi$ and $\sigma^{-}$light, atoms in the trapped state are off-resonantly driven to excited states that can decay to nontrapped ground states. Most of the population transfer is to the $2 S_{1 / 2}\left(m_{J}=-\frac{1}{2}, m_{I}=\frac{3}{2}\right)$ state and, to a lesser extent, to the $2 S_{1 / 2}\left(m_{J}=\frac{1}{2}, m_{I}=\frac{1}{2}\right)$ state. Fortunately, the trap laser beams repump the majority of the $2 S_{1 / 2}\left(m_{J}=-\frac{1}{2}, m_{I}=\frac{3}{2}\right)$ atoms back into the trapped state before they can escape. Atoms in states with $m_{I}=\frac{1}{2}$ are not effectively repumped so their production is the principal loss mechanism during loading. The calculated loss rate for this channel is consistent with the measured loss rate of 1.5 $\mathrm{s}^{-1}$. This rate may be minimized by optimization of the trap beam polarizations and through the use of additional repumping laser beams.

After loading the atoms into the trap, the trap beams can be turned off, leaving the atoms in the dark. The lifetime of atoms in the dark is $240 \mathrm{~s}$, presumably limited by collisions with background gas in the trap vacuum chamber that operates at an estimated pressure of $1 \times 10^{-11}$ Torr.

The temperature of the trapped atoms is estimated by their excitation line shape, measured from fluorescence induced by weak probe laser beams. Probing is provided by the same laser beams used to load the trap, but with substantial attenuation and variable detuning from the frequency used for loading. By varying the probe detuning, atoms at different magnetic fields are resonantly excited [12]. In the trap, the Zeeman shift experienced by an atom is proportional to its potential energy, so that the excitation line shape describes the energy distribution of the trapped atoms. We model the line shape by assuming a Boltzmann energy distribution for the trapped atoms and a harmonic trap potential [12]. The measured excitation line shape of the trapped atoms is shown in Fig. 3, where it is compared to the model using the best-fit temperature of $1.1 \mathrm{mK}$. The uncertainty in the fit is \pm 0.1 $\mathrm{mK}$. The relatively high temperature is due to the large intensity of the trap beams required for efficient loading. By lowering the trap beam intensity at the end of loading, it should be possible to reduce the temperature to near the Doppler limit of $140 \mu \mathrm{K}$, which would increase the peak trap 


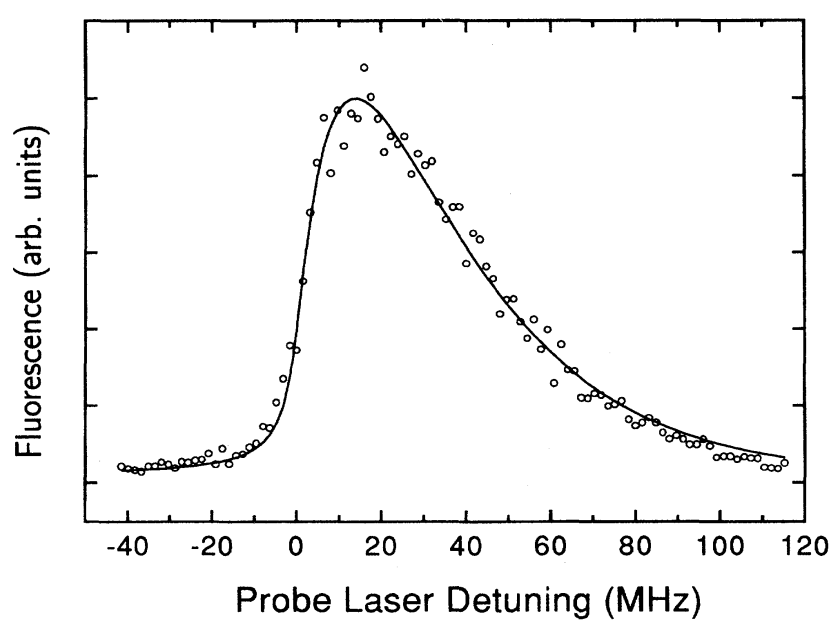

FIG. 3. Excitation line shape of trapped lithium atoms. The data are given by the circles and the solid line represents a model which assumes a Boltzmann energy distribution for atoms in a harmonic potential. The best-fit temperature for the trapped atoms is $1.1 \mathrm{mK}$, which together with the number of atoms indicates a peak density of approximately $3 \times 10^{9} \mathrm{~cm}^{-3}$.

density by a factor of 20 . The number of atoms in the trap is determined by imaging the fluorescence induced by the trap beams onto a photomultiplier tube. Given the distribution of atoms in the trap, the average scattering rate induced by the trap beams can be calculated. This rate, together with the estimated light collection and detection efficiency, indicates that the equilibrium number of atoms is approximately $1 \times 10^{8}$, to within a factor of 2 uncertainty.

The trapped-atom temperature, number of trapped atoms, and the shape of the potential determine the density distribution. Assuming a harmonic trap potential, the measured number and temperature imply a peak density of approximately $3 \times 10^{9} \mathrm{~cm}^{-3}$. This density is consistent with absorption measurements obtained by focusing a weak, resonant laser beam through the center of the trapped-atom cloud.

While loading the trap, the number of trapped atoms is determined by the balance of the loading rate and the total loss rate. Flux measurements of the $1-\mathrm{cm}$-diam cold-atom beam entering the trap chamber indicate a trap loading efficiency of a few percent. We believe the loading efficiency to be limited by the range of velocities that can be adequately damped and by the spatial acceptance of the trap. Improvements in loading may be made by spatially compressing the cold atomic beam, using, for example, magneto-optical compression [13] or by optimizing the slow atom-beam velocity distribution.

The density of the trapped atoms is partially determined by the shape of the confining potential. For a given number of atoms in the trap at a given temperature, tighter confinement will result in higher density. We initially built a trap that was ten times smaller than the present one, which had a bias field of $1955 \mathrm{G}$, a depth of $500 \mathrm{G}$, and harmonic field coefficients of $d^{2} B / d x^{2}=d^{2} B / d y^{2}=350 \mathrm{kG} / \mathrm{cm}^{2}$ and $d^{2} B / d z^{2}=50 \mathrm{kG} / \mathrm{cm}^{2}$. Attempts to load this trap were unsuccessful for several possible reasons. The larger field gradients reduced the range of velocities that could be sufficiently damped to permit trapping. In addition, the spatial acceptance of the trap was reduced, both because of the smaller trap size and because of larger fringing field gradients which could deflect incident slow atoms.

With modest improvements in temperature and loading we expect to achieve trapped-atom densities greater than $10^{10} \mathrm{~cm}^{-3}$. The recently obtained $s$-wave elastic ${ }^{7} \mathrm{Li}-{ }^{7} \mathrm{Li}$ scattering cross section of $5.0 \times 10^{-13} \mathrm{~cm}^{2}$ [14] and the trap lifetime suggest that at these densities evaporative cooling should be possible using radio-frequency or optical radiation to selectively remove the most energetic atoms [7,15]. A density of $10^{13} \mathrm{~cm}^{-3}$ is needed to reach the conditions for observing Bose-Einstein condensation at a temperature of $1 \mu \mathrm{K}$. For the present trap, this would require $10^{7}$ atoms.

It has been demonstrated that neutral atoms can be loaded and trapped in the nonzero minimum of the magnetic field generated by an Ioffe configuration of permanent magnets. Permanent magnets can produce extremely high field gradients that tightly confine the atoms. The use of permanent magnets provides trap fields and gradients similar to those achieved with superconducting magnets but with a more open design, allowing greater optical access. The bias field eliminates the possibility of motion-induced spin-flip transitions that can occur in traps that contain a zero in magnetic field. The trapped-atom density can be increased by further cooling to the point where quantum-statistical effects may be observed.

This work is supported by the National Science Foundation, the Welch Foundation, and the Texas Advanced Technology Program. C.A.S. acknowledges the support of the NSF.
[1] See special issues on laser cooling and trapping: J. Opt. Soc. Am. B 6 (1989); Laser Phys. 4 (1994).

[2] J. J. Tollett, C. C. Bradley, and R. G. Hulet, Bull. Am. Phys. Soc. 37, 1126 (1992); V. Frerichs, W. G. Kaenders, and D. Meschede, Appl. Phys. A 55, 242 (1992).

[3] V. Bagnato, D. E. Pritchard, and D. Kleppner, Phys. Rev. A 35, 4354 (1987).

[4] A. Höpe, D. Haubrich, G. Müller, W. G. Kaenders, and D.
Meschede, Europhys. Lett. 22, 669 (1993).

[5] Y. V. Gott, M. S. Ioffe, and V. G. Tel'kovskii, Nucl. Fusion, 1962 Suppl., Pt. 3, 1045 (1962); T. Bergeman, G. Erez, and H. J. Metcalf, Phys. Rev. A 35, 1535 (1987); V. S. Bagnato, G. P. Lafyatis, A. G. Martin, E. L. Raab, R. N. Ahmad-Bitar, and D. E. Pritchard, Phys. Rev. Lett. 58, 2194 (1987).

[6] D. E. Pritchard, Phys. Rev. Lett. 51, 1336 (1983); A. L. Migdall, J. V. Prodan, W. D. Phillips, T. H. Bergeman, and H. 
J. Metcalf, ibid. 54, 2596 (1985).

[7] W. Petrich, M. H. Anderson, J. R. Ensher, and E. A. Cornell (unpublished).

[8] T. E. Barrett, S. W. Dapore-Schwartz, M. D. Ray, and G. P. Lafyatis, Phys. Rev. Lett. 67, 3483 (1991).

[9] J. Nellessen, J. H. Müller, K. Sengstock, and W. Ertmer, J. Opt. Soc. Am. B 6, 2149 (1989).

[10] V. I. Balykin, V. S. Letokhov, and A. I. Sidorov, Pis'ma Zh. Eksp. Teor. Fiz. 40, 251 (1984) [JETP Lett. 40, 1026 (1984)].

[11] S. Chu, L. Hollberg, J. E. Bjorkholm, A. Cable, and A. Ashkin, Phys. Rev. Lett. 55, 48 (1985).

[12] A. G. Martin, K. Helmerson, V. S. Bagnato, G. P. Lafyatis, and
D. E. Pritchard, Phys. Rev. Lett. 61, 2431 (1988); K. Helmerson, A. Martin, and D. E. Pritchard, J. Opt. Soc. Am. 9, 483 (1992).

[13] E. Riis, D. S. Weiss, K. A. Moler, and S. Chu, Phys. Rev. Lett. 64, 1658 (1990); J. Nellessen, J. Werner, and W. Ertmer, Opt. Commun. 78, 300 (1990).

[14] E. R. I. Abraham, W. I. McAlexander, C. A. Sackett, and R. G. Hulet (unpublished).

[15] I. D. Setija, H. G. C. Werij, O. J. Luiten, M. W. Reynolds, T. W. Hijmans, and J. T. M. Walraven, Phys. Rev. Lett. 70, 2257 (1993); K. B. Davis, M.-O. Mewes, M. A. Joffe, and W. Ketterle (unpublished). 


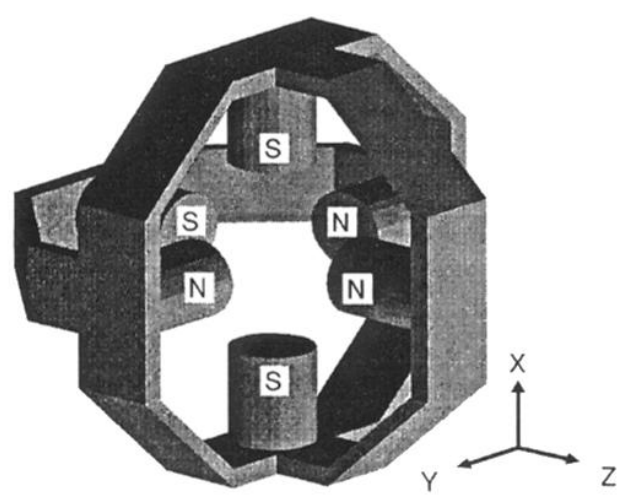

FIG. 1. Diagram showing the orientation and placement of the cylindrical trap magnets for the permanent magnet, Ioffe configuration trap. The letters indicate the inner tip magnetizations of the $\mathrm{Nd}-\mathrm{Fe}-\mathrm{B}$ cylinder magnets, $\mathrm{N}$ for north and $\mathrm{S}$ for south. The tip-totip magnet spacing is $4.45 \mathrm{~cm}$. The structure around the magnets is an iron yoke that supports the magnets and provides low reluctance paths for the flux to follow between magnets of opposite sign. 